\title{
Giant Pulses from the Crab Pulsar
}

\author{
Timothy H. Hankins \\ Physics Department, New Mexico Institute of Mining and Technology, \\ Socorro, NM 87801 USA : thankins@nrao.edu
}

\begin{abstract}
Recent observations of the "giant" radio pulses received from the Crab pulsar are reviewed. The frequency range over which they have been detected spans at least two decades, and individual pulses appear to have a frequency span of $3: 1$ or more. The pulse structure is dominated by scattering at frequencies below about $2 \mathrm{GHz}$; at higher frequencies the intrinsic pulse width is about $1 \mu$ s with nanostructure extending down to $10 \mathrm{~ns}$. The polarization structure may be contaminated by scattering at low frequencies and the polarization is weak and highly variable at high frequencies. No definitive emission mechanism with testable hypotheses for the giant pulses has been put forth. One of the most promising, based on the collapse of plasma solitons, predicts nanostructure bandwidths narrower than what is seen for the giant pulses themselves, though the observations do not show wideband correlation of the shortest resolvable intensity structure.
\end{abstract}

\section{Introduction}

The Crab pulsar emits occasional pulses which can be thousands of times more intense than the average pulse. In fact, the Crab pulsar was first discovered by these giant pulses, rather than by the periodic pulse train by which most pulsars are found. Staelin \& Reifenstein (1968) recorded several giant pulses in October 1968 using a multichannel filter bank to generate a dynamic spectrum; the spectral tracks of the giant pulses received at $112 \mathrm{MHz}$ were quite distinctive of a pulse dispersed by propagation through the interstellar medium.

\section{Occurrence}

At frequencies below $2 \mathrm{GHz}$ where the correlation bandwidth imposed by diffractive scintillation is small, the giant pulses can be seen in approximately $1 \%$ of pulse periods with a frequency-dependent power-law flux distribution (Lundgren 1994, Shrauner 1997). Giant pulses occur at the pulse phase of the main pulse and less often at the interpulse phase, but never at the phase of the precursor, nor at the phase of the anomalous high-frequency average profile components detected at 1.4, 4.8, and $8.4 \mathrm{GHz}$ (Moffett and Hankins 1996). The giant pulses can occur anywhere within the phase window defined by the average pulse. At high frequencies the pulses are much narrower than the average profile, and the arrival times jitter randomly from one occurrence to the next. 
There is no correlation of giant pulses from one period to the next, although I have seen giant pulses in consecutive periods. Figure 1 shows a pair of giant pulses recorded at $333 \mathrm{MHz}$ which occur in the same pulse period.

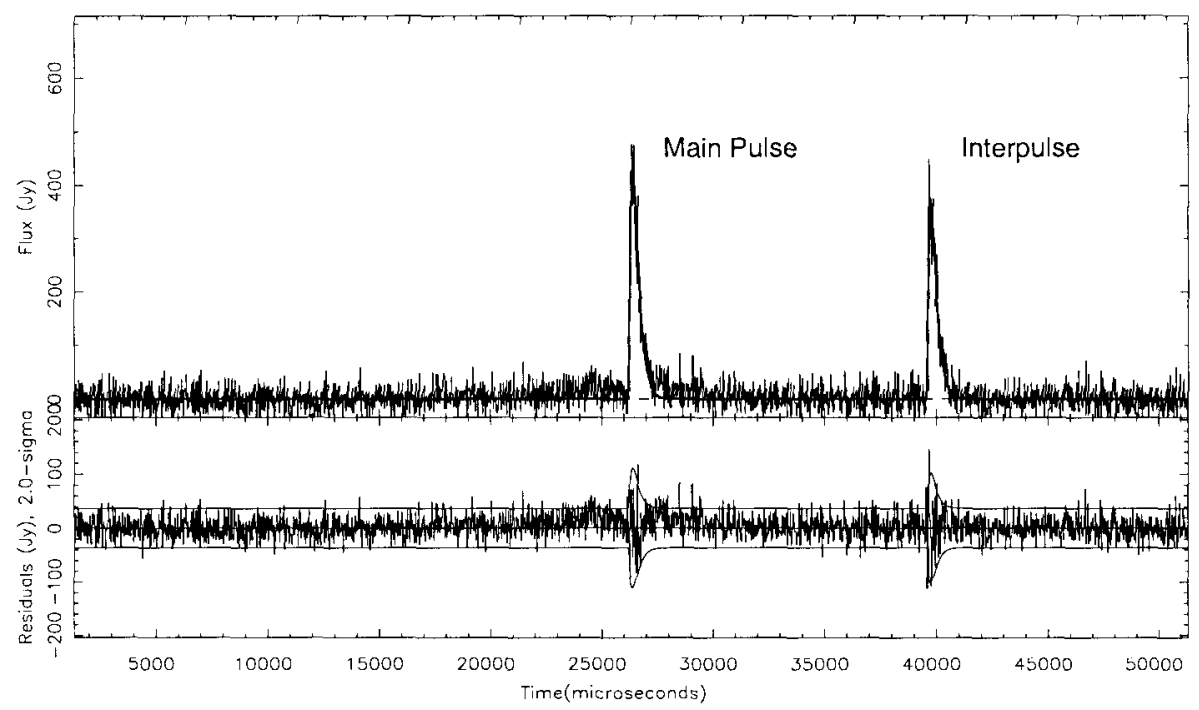

Figure 1. A giant pulse occurring at the phase of the main pulse, followed 14 ms later by a giant interpulse, recorded at $0.333 \mathrm{GH} \%$ is shown . The total intensity is fitted by an "xfred" (Fast-Rise, Exponential Decay) function $a_{1}\left(t-a_{0}\right) e^{-\left(t-a_{0}\right) / a_{2}}$. The lower trace shows the $2 \sigma$ estimation error of the fit and the fit residuals. Both the main pulse and interpulse have approximately the same width, as would be expected from scattering broadening.

\section{Spectra}

Crab giant pulses were discovered near $0.1 \mathrm{GHz}$, and I have detected one pulse in several hours observing at $15 \mathrm{GH} \%$. Their occurrence above $2 \mathrm{GHz}$ appears to be strongly affected by ISS; during most observing sessions the giant pulses are not seen at 4.8 nor at $8.4 \mathrm{GHz}$, yet on two occasions at $8.4 \mathrm{GHz}, 30$-second bursts of pulses occurred, strong enough to see pulses in virtually every period.

Several studies of the spectra have been made. As a test of the bandwidth and simultaneity of giant pulses, simultaneous observations at widely separated frequency pairs have shown that, after correction for the interstellar dispersion delay, giant pulses at different frequencies occur at the same time (Sallmen et al., 1999) over a frequency range of at least 3:1. Figure 2 shows the auto- and cross correlations of a pulse recorded at both 4.885 and $1.435 \mathrm{GHz}$ at the VLA. This pair of pulses was selected because of their similarity at the two frequencies; more often the pulses shapes are considerably different at these two frequencies, although their arrival times are consistent with only dispersion delay between 

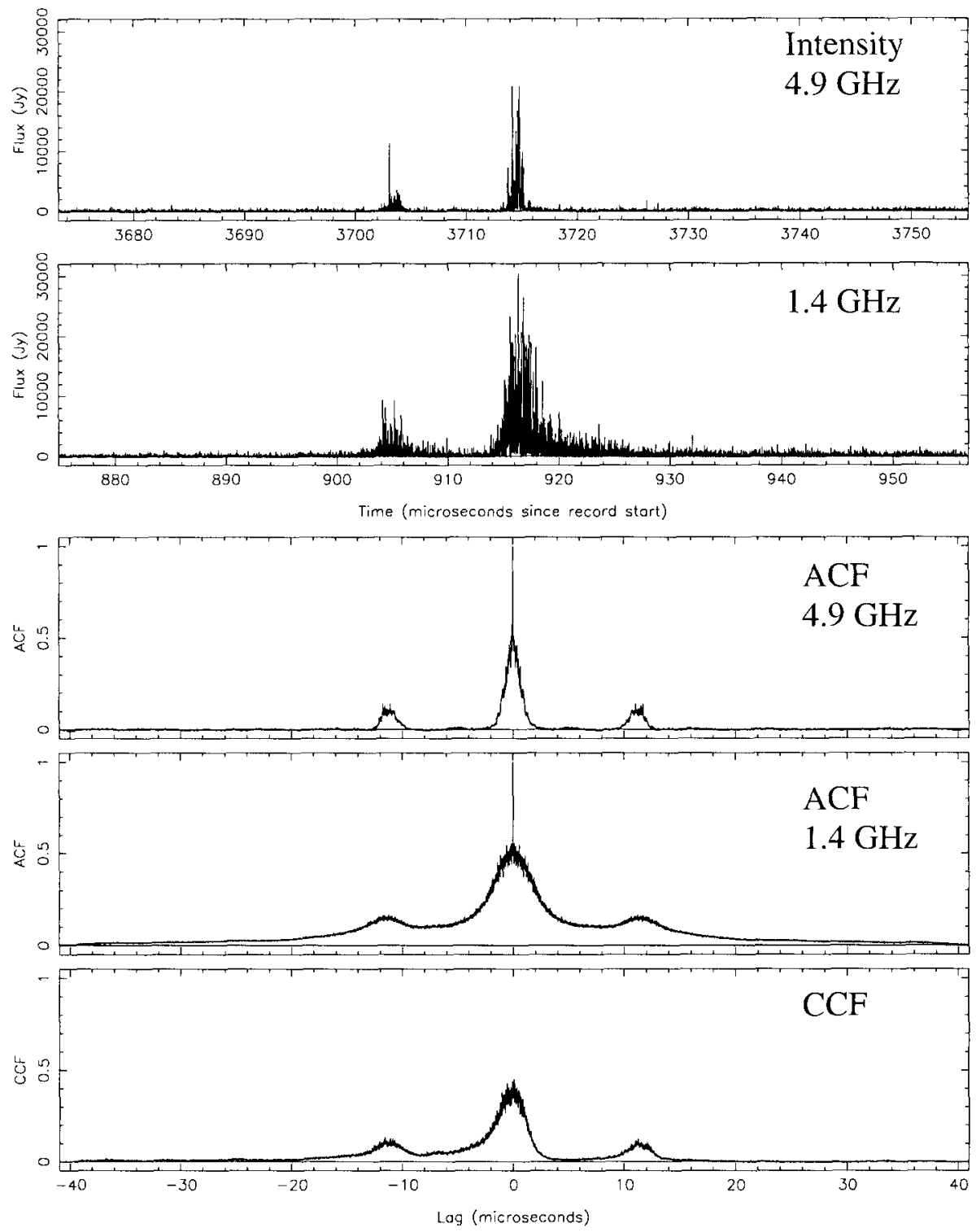

Figure 2. A single giant pulse recorded at 4.885 and $1.435 \mathrm{GHz}$ is shown with the dispersion delay removed, plus the normalized autocorrelation function (ACF) and cross-correlation function (CCF) with the appropriate corrections for the Amplitude Modulated Noise Model (Rickett 1975). The lower frequency ACF is clearly broadened by ISS.

them. 'The spectral indices obtained from these observations range from -2.2 to -4.9 with an average of -3.4 between 0.6 and $1.4 \mathrm{GHz}$, (Sallman et al., 1999) 
and between 0 and -4 from 1.4 and $4.8 \mathrm{GHz}$ (Moffett 1997). A histogram of the spectral indices for the latter observations is shown in Figure 3. The interpulse histogram is very similar; both show a distribution peak between -1.5 and -2.0 . The spectral index for stronger pulses appears to be steeper than for weaker pulses, but this may be an artifact of the pulse selection process.

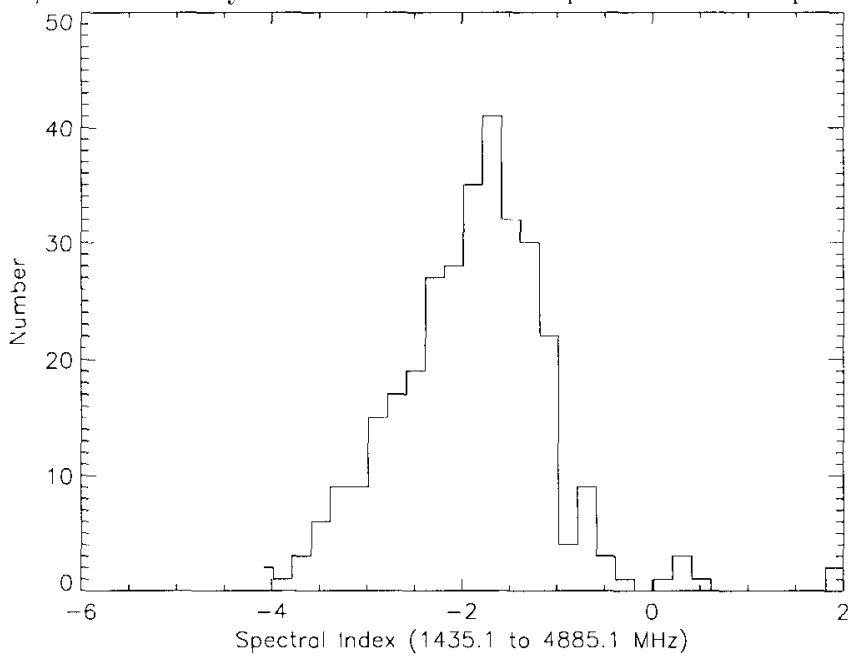

Figure 3. Giant mainpulse spectral index histogram, 1.4 to $4.9 \mathrm{GHz}$.

\section{Pulse shapes}

At frequencies below about $1 \mathrm{GHz}$ the giant pulse shapes are dominated by scattering broadening which increases with wavelength as expected from interstellar scattering. At $1.4 \mathrm{GHz}$ subpulse structure is seen in some pulses which may be intrinsic or may result from nebular scattering near the pulsar (Cordes 1998). A sequence of four giant pulses recorded within one minute are shown in Figure 4. Each of these pulses has a different number of components, but they are all reasonably well fitted by the "xfred" function given in the caption of Figure 1.

The pulses are considerably shorter at $4.8 \mathrm{GHz}$, showing durations less than $1 \mu \mathrm{s}$, but some of these pulses continue to show the "xfred" shape but with a value of $a_{2}$ which scales from $1.4 \mathrm{GHz}$ as $f^{-1.9 \pm 0.2}$. Pulses with structure which is not resolved at our highest resolution of $10 \mathrm{~ns}$ are occasionally found at both 4.8 and $8.4 \mathrm{GHz}$. The shape of the single pulse detected at $15 \mathrm{GHz}$ is consistent with the shapes seen at $8.4 \mathrm{GHz}$, i.e., width of about $1 \mu \mathrm{s}$ with shorter modulation.

\section{Emission Mechanism Constraints}

The collapsing soliton model of Weatherall (1998) predicts an instantaneous bandwidth of about $20 \%$ for the shortest ( $1 \mathrm{~ns}$ ) structure. To test this bypothesis we conducted a set of simultaneous dual-frequency observations at the VLA with frequency separations of 0.05 to $3.4 \mathrm{GHz}$. The pulse intensities recorded at both 

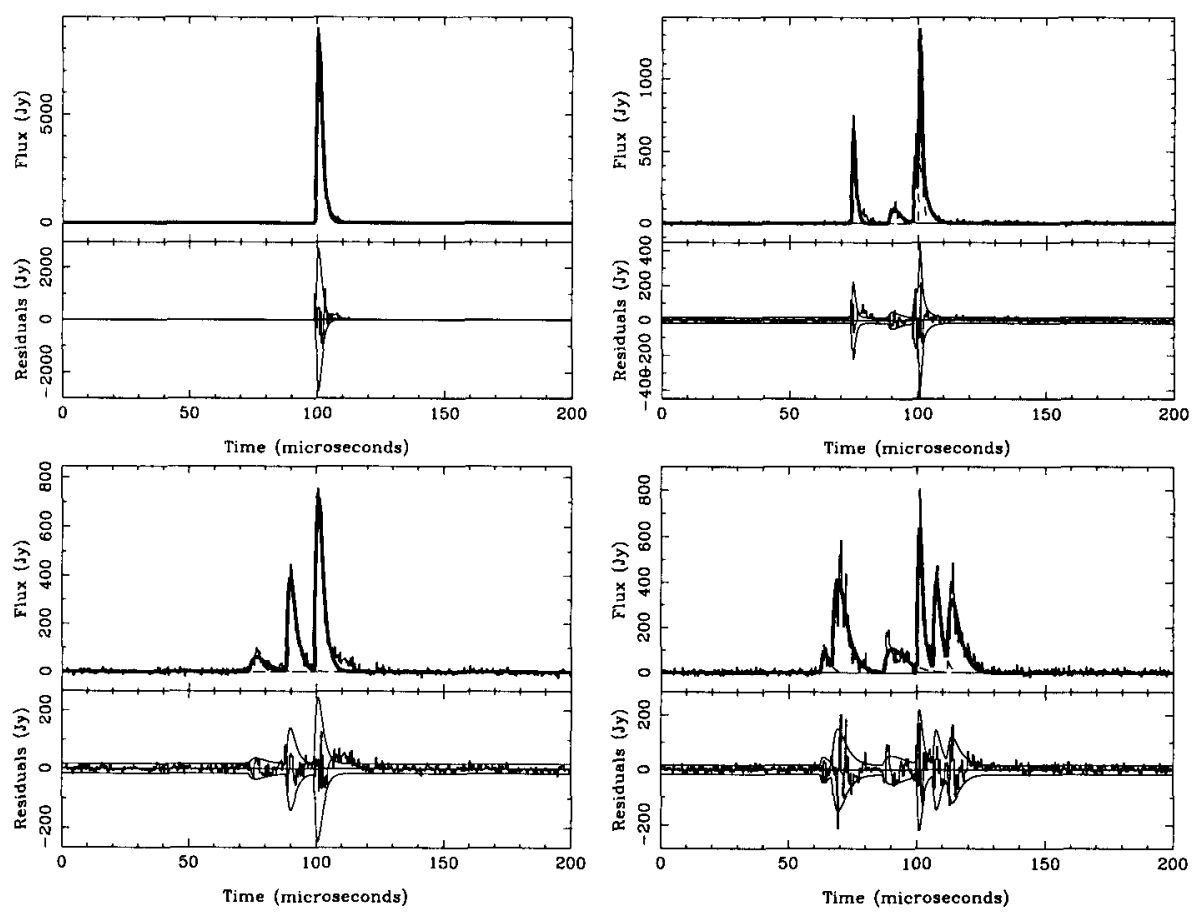

Figure 4. Four giant pulses all recorded within one minute at $1.4 \mathrm{GHz}$ are shown, with "xfred" fits and residuals.

frequencies were cross-correlated; if there is detectable, unresolved wideband structure, then it should appear as a "spike" in the cross-correlation (CCF), much like the zero-lag spike expected in the autocorrelations as predicted by Rickett's Amplitude Modulated Noise Model (Rickett 1995), but no such feature is seen. In Figure 5 the ACFs of the total intensity at 4.985 and $4.535 \mathrm{GHz}$ and their CCF are shown. The ACFs show the expected zero-lag spike, and the CCFs show that although the individual pulse envelopes are well correlated, there is no unresolved structure that appears at both frequencies.

Acknowledgments. This work on the Crab pulsar giant pulses has involved a number of people, including Don Backer, Tracey Delaney, Jean Eilek, Jeff Kern, Scott Lundgren, David Moffett, Shauna Sallmen, and Jim Weatherall, plus the NRAO staff at the VLA. It has been supported by NSF grants AST9315285 and AST-9618408. The National Radio Astronomy Observatory is a facility of the National Science Foundation operated under cooperative agreement by Associated Universities, Inc.

\section{References}

Cordes, J. M. 1998, personal correspondence

Lundgren, S. C. 1994, PhI) Thesis, Cornell University 

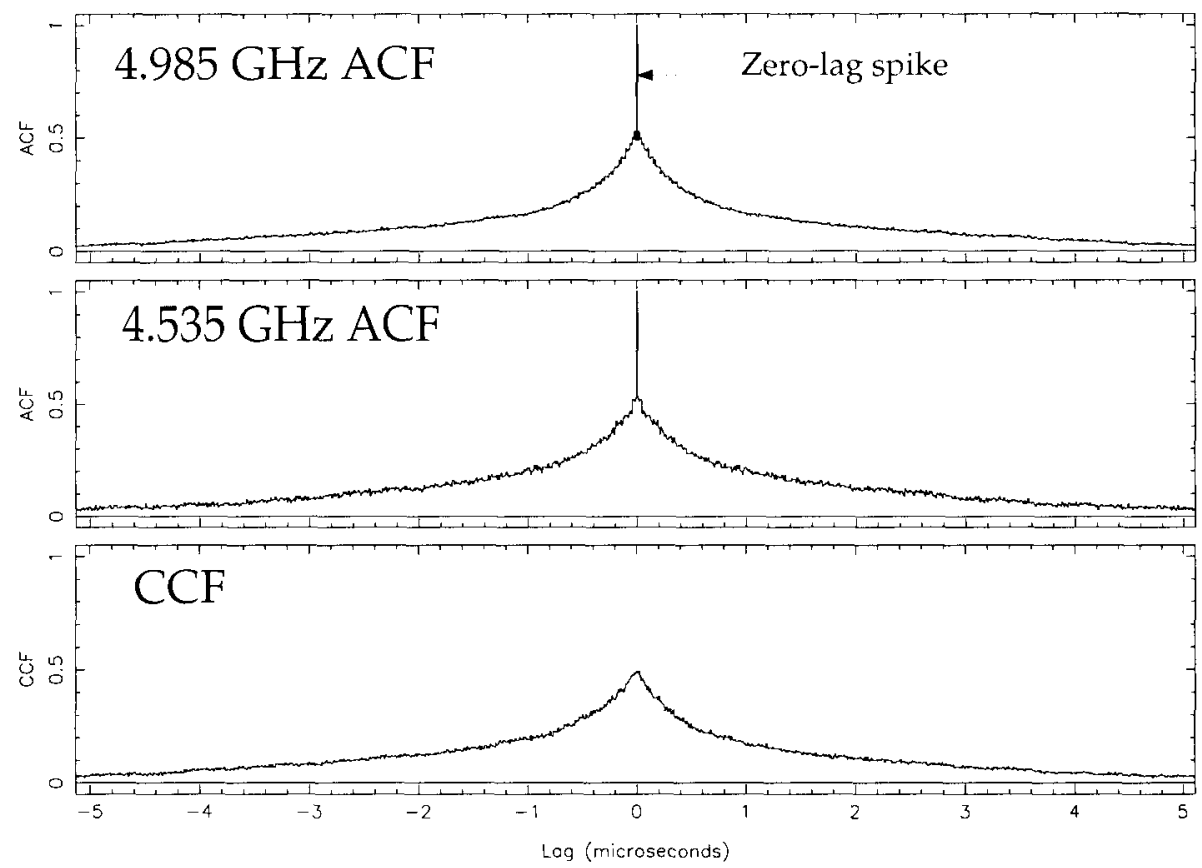

Figure 5. The $\mathrm{ACF}$ and $\mathrm{CCF}$ of the total intensity recorded at 4.985 and $4.535 \mathrm{GHz}$ are shown. The sample rate of $100 \mathrm{MHz}$ gives an unsmoothed resolution of $10 \mathrm{~ns}$. The dispersion delay has been subtracted from the CCF. The individual pulses contain such short structure that the dispersion measure (DM) can be determined to better than $0.001 \mathrm{pc} \mathrm{cm}^{-3}$. For the data shown a trial DM was used initially for coherent dedispersion processing. The DM was subsequently refined from the CCF and the data then reprocessed with the improved DM value of $56.7990 \mathrm{pc} \mathrm{cm}^{-3}$ for $20 \mathrm{Dec} 1998$.

Moffett, D. A. 1997 PhD Thesis, New Mexico Institute of Mining and Technology Moffett, D. A. \& Hankins, 'T. H. 1996, ApJ, 468, 779

Rickett, B. J. 1995, ApJ, 197, 185

Sallmen, S., Backer, D. C., Hankins, T. H., \& Lundgren, S. 1999, Ap.J, 517, 460 Shrauner, J. A. 1997, PhD Thesis, Princeton University

Staelin, D. H. 1968, \& Reifenstein, III, E. C., Science, 162, 1482

Weatherall, J.C., 1998, ApJ, 506, 341 